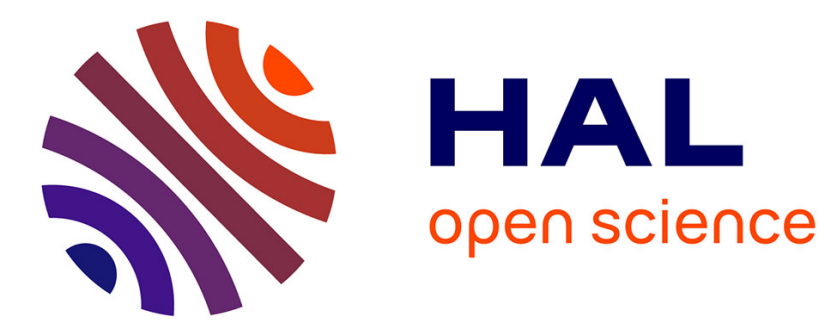

\title{
A parallel unbalanced digitization architecture to reduce the dynamic range of multiple signals
}

\author{
Mathieu Vallerian, Florin Doru Hutu, Guillaume Villemaud, Benoit
}

Miscopein, Tanguy Risset

\section{- To cite this version:}

Mathieu Vallerian, Florin Doru Hutu, Guillaume Villemaud, Benoit Miscopein, Tanguy Risset. A parallel unbalanced digitization architecture to reduce the dynamic range of multiple signals. Radio Science, 2016, 10.1002/2015RS005885 . hal-01312986

\section{HAL Id: hal-01312986 \\ https://hal.science/hal-01312986}

Submitted on 2 Jun 2016

HAL is a multi-disciplinary open access archive for the deposit and dissemination of scientific research documents, whether they are published or not. The documents may come from teaching and research institutions in France or abroad, or from public or private research centers.
L'archive ouverte pluridisciplinaire HAL, est destinée au dépôt et à la diffusion de documents scientifiques de niveau recherche, publiés ou non, émanant des établissements d'enseignement et de recherche français ou étrangers, des laboratoires publics ou privés. 


\title{
A parallel unbalanced digitization architecture to reduce the dynamic range of multiple signals
}

\author{
Mathieu Vallérian, ${ }^{1,2}$ Florin Huţu ${ }^{2}$, Guillaume Villemaud, ${ }^{2}$ Benoît Miscopein ${ }^{3}$ and \\ Tanguy Risset ${ }^{2}$
}

Key Points.

$\circ$ An radio-frequency architecture able to treat high dynamic range signals is proposed

- The proposed architecture allowed to release the analog-to-digital constraints

- The architecture is validated through system simulations

Accepted for publication in Radioscience. Copyright 2016 American Geophysical Union. Further reproduction or electronic distribution is not permitted.

Technologies employed in urban sensor networks are permanently evolving and thus the gateways employed to collect data in such kind of networks have to be very flexible in order to be compliant with the new communication standards. A convenient way to do that is to digitize all the received signals in one shot and then to digitally perform the signal processing, as it is done in Software-Defined Radio (SDR). All signals can be emitted with very different features (bandwidth, modulation type and power level) in order to respond to the various propagation conditions. Their difference in terms of power levels is a problem when digitizing them together, as no current commercial Analog-to-Digital Converter (ADC) can provide a fine enough resolution to digitize this high dynamic range between the weakest possible signal in presence of a stronger signal. This paper presents an RF front-end receiver architecture capable of handling this problem by using two ADCs of lower resolutions. The architecture is validated through a set of simulations using Keysight's ADS software. The main validation criteria is the BER comparison with a classical receiver.

\section{Introduction}

In 2010, the number of physical objects connected to the Internet had surpassed the number of living humans on Earth. Moreover, in the next years, we will assist to an exponential increase of this kind of devices with 212 billion IoT smart objects expected in 2020 [Gantz and Reinsel, 2012]. Together with this increase, several challenges are emerging, such as energy consumption reduction, the

\footnotetext{
${ }^{1}$ Orange Labs, 38243, Meylan, France.

${ }^{2}$ Univ Lyon, INSA Lyon, Inria, CITI, F-69621

Villeurbanne, France.

${ }^{3}$ CEA, LETI, Minatec campus, F-38054, Grenoble, France
}

Copyright 2016 by the American Geophysical Union. 0048-6604/16/\$11.00 amount of big data management and the hardware capability to be flexible and to adapt to new communication standards on the fly. From the RF telecommunications perspective, the last aspect cited here is very challenging since it may imply the transfer of all the signal processing in the digital domain and the use of "universal" RF frontends like in Software-Defined Radio (SDR) [Dardaillon et al., 2014].

Still, the main problem is the dynamic range of magnitudes of the received signals which imposes hard constraints on the analog-to-digital conversion stage, both in terms of dynamic range and of bandwidth. In order to relax these constraints, this paper proposes the use of an RF front-end with two digitization branches which separates the highest amplitude received signal from the low amplitude ones. With the cost of supplementary RF hardware, 
two low resolution ADCs will be used instead of using one high resolution ADC. In order to validate the proposed architecture, which is dedicated to applications of urban sensor networks, realistic values for the bandwidth, modulation type and signal dynamic range are defined.

A common urban sensor network is composed of sensor nodes and sink nodes, also called gateways. The sensor nodes collect the data and send it to the sink node. They are usually Short Range Devices (SRDs) that emit signals around $868 \mathrm{MHz}$ in an $8 \mathrm{MHz}$ transmission bandwidth [ETSI, 2011].

Sensor nodes are used in numerous applications, such as water and gas metering, parking management, air quality monitoring, etc. Each application has its own constraints, and thus the sensors emit with significantly different propagation conditions (some sensors being underground, some other being in line-of-site conditions). To overcome these constraints the sensors use different communication protocols, thus the signals received by the gateway may have different features.

In a classical gateway, the different technologies are implemented by stacking up the receivers, each receiver being dedicated to a certain communication protocol. This approach has limitations because new technologies are still emerging today. Consequently, the gateway should be designed in order to adapt itself to these new transmission technologies. As mentioned previously, one of the envisaged solutions is to perform the signal processing as much as possible in the digital domain. To do this, the $8 \mathrm{MHz}$ transmission bandwidth, commonly employed in urban sensor networks, can be digitized at once to guarantee a high flexibility, as done in SoftwareDefined Radio (SDR) systems [Dardaillon et al., 2014]. Then, a new technology could be embedded in the gateway only via a software update.

In [Vallerian et al., 2014], it has been shown that, considering the various technologies employed and the various propagation conditions, the gateway should be able to receive and demodulate signals having a power ratio up to $100 \mathrm{~dB}$, otherwise, too many messages would be lost. This high power ratio poses a problem as the ADCs should have a high enough resolution for that.

This issue is illustrated in Figure 1, where two signals (one strong and one weak, i.e. one with a high power level and one with a low power level) are represented as sine functions for convenience. When the signals are received together, the dynamic range of the total signal is driven by the strong signal's dynamic range. Thus on the ADC's full resolution, only a few bits are used to digitize

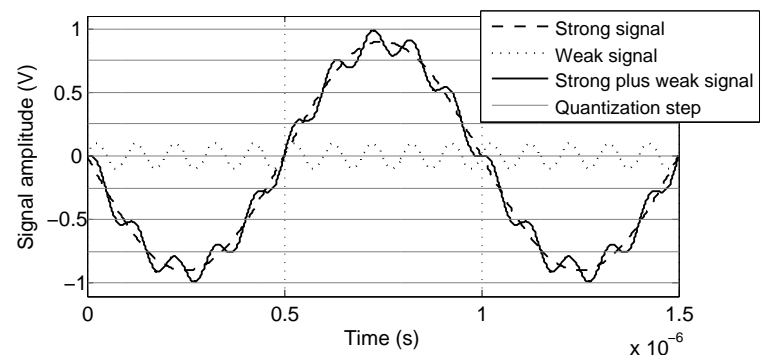

Figure 1. A strong and a weak signal being digitized together. When they are combined, the quantization noise level is higher than the weak signal level, leading to its degradation.

the weak signal. Moreover, the required ADC resolution to digitize the weak signal increases with the power ratio between the signals.

With a $100 \mathrm{~dB}$ power ratio and a $8 \mathrm{MHz}$ bandwidth, it has been shown in [Vallerian et al., 2014] that an ADC of at least 21 bits is required and, to the best of the authors' knowledge, such a resolution is not affordable with today's ADC. Typically, ADCs providing high resolution are used in audio processing with a resolution of 24 bits for only $192 \mathrm{kHz}$ of bandwidth. With an $8 \mathrm{MHz}$ bandwidth, one cannot reasonably expect a higher resolution than 16 bits [Jonsson, 2010]. In this context, this paper presents an architecture aiming at relaxing the ADC constraints by using two digitization branches.

This architecture is conceived based on the assumption that only one strong signal is present in the transmission band, whereas there can be several weak signals. Indeed a strong signal always comes from a sensor that is located near the gateway, generally in Line-of-Sight (LOS) propagation conditions, whereas a weak signal may come from various locations. Moreover, in urban sensor networks, the nodes emit essentially short frames, so the interference probability is very low in the case of strong signals since only a small number of nodes is able to provide such a strong signal [ETSI, 2011].

This paper is structured as follows: section 2 introduces the proposed architecture and in section 3, the implementation of this architecture on the Keysight's ADS software is described. Then the simulation results, characterizing the architecture robustness on the hardware implementation impairments are given in section 4. Section 5 concludes this paper and gives directions for future work. 


\section{Architecture description}

\subsection{Motivation and state of the art}

It has been shown in [Vallerian et al., 2014] that the dynamic range occupied by the various signals in our scenario of urban sensor networks is too high to properly digitize the whole band at once. Then, one solution to keep a full band digitization is to reduce this dynamic range, either by amplifying the small signals or by attenuating the strong ones. To choose the best approach, one can consider that a strong signal always comes from a sensor that is located near the gateway in LOS propagation conditions, whereas a weak signal may come from a far wider area, as some sensors are located far from the gateway, underground or, more globally, in Non-Line-ofSight (NLOS) propagation conditions. Then it is more likely to receive a weak signal than a strong signal.

As a consequence, it is assumed here that there will be one strong signal co-existing with several weak signals. Hence, it seems more relevant to attenuate the strong signal than to amplify the weak signals.

Figure 2 shows the required resolution when demodulating two signals with different power levels ([Vallerian et al., 2014]). Assuming as a target that a resolution of 16 bits is feasible, the dynamic range of the signals should be reduced to a maximum of $70 \mathrm{~dB}$. If a margin of 2 bits is taken between the ADC's resolution and its Effective Number Of Bits (ENOB), then the strong signal should be attenuated by $40 \mathrm{~dB}$. The saving of resolution bits and the dynamic range reduction are highlighted in Figure 2 with dashed lines.

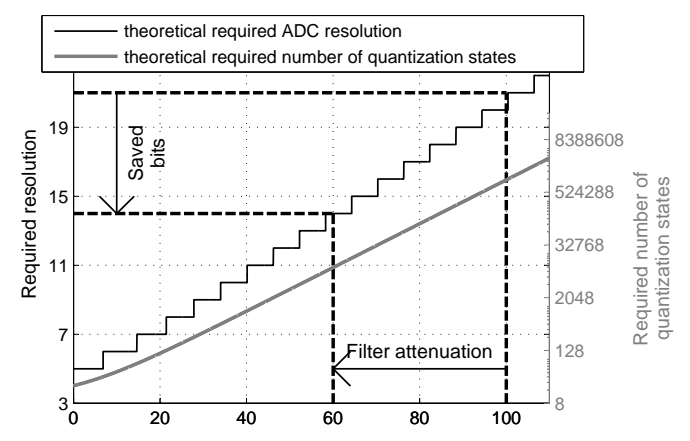

Figure 2. The required resolution to properly demodulate two signals with high dynamic range. 21 bits are required to digitize the signals with a dynamic range of $100 \mathrm{~dB}$, but the required resolution can be reduced to 14 bits if the dynamic range is reduced by $40 \mathrm{~dB}$.
The problem addressed in this paper may seem to be similar to the one of interference cancellation ([Borremans et al., 2011; van den Heuvel and Cabric, 2010]). However, in these works, it is always assumed that the interference must be totally eliminated, whereas in our case it is also a signal of interest. In [Wang et al., 2005], an architecture is presented to improve the near-far performance in Code Division Multiple Access (CDMA). However, it requires to know the signals signatures and thus it requires a minimum number of previously received signals. Moreover, the performance is discussed by the authors for a power ratio of only 10 between the signals.

Another architecture, using a Minimum Mean-Square Error (MMSE) filter and taking advantage of both successive interference cancellation (SIC) and parallel interference cancellation (PIC), was proposed in [Krzymien and Schlegel, 2011]. However, the considered maximum dynamic range is of the order of tens of $\mathrm{dB}$ and is lower than the one in our scenario.

Another way to reduce the dynamic range can be to subtract the strong signal from the received signal, as proposed in [Nie et al., 1999]. This method can bring some instability to the system, as the subtracted signal is generated through a prediction algorithm. Indeed prediction algorithms are intended for slowly-varying signals [Fletcher et al., 2007], whereas in our case the dynamic range can be very high.

In this paper, the proposed solution is to use a notch filter that attenuates the strong signal and leaves the rest of the transmitted band unchanged. The main challenge with this approach is to attenuate the strong signal regardless of its frequency while preserving the rest of the band.

\subsection{Receiver's architecture description}

The proposed architecture is depicted in Figure 3 and has a notch filter to attenuate the strong signal. Two solutions are possible to reconfigure this receiver's architecture in order to properly attenuate the strong signal: either the notch filter's central frequency can be modified to match the strong signal's central frequency, or the strong signal's carrier frequency can be translated to match the notch filter's central frequency.

With the first solution, a reconfigurable filter should be used and its stop-band should be $8 \mathrm{MHz}$ wide. With the second solution, the strong signal should be translated into an intermediate frequency that is equal to the notch filter's central frequency and translated in baseband afterward. In both cases, the strong signal's central frequency must be evaluated before the reconfiguration. 


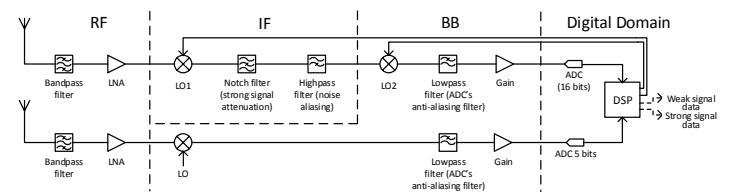

Figure 3. Schematic of the proposed architecture. The Coarse Digitization Path (on bottom) allows to digitize the strongest signal. Its frequency is digitally found, and then the signals are down converted on the Fine Digitization Path (on top) in order to attenuate the strongest signal by means of a notch filter, reducing the dynamic range of the signals.

The second solution (with a fixed filter) seems simpler to implement, as it only requires two mixers to perform the frequency translations. With the first method, a reconfigurable filter with a high enough selectivity to attenuate the strong signal without affecting the rest of the band is required. The filter's non-reconfigurability is thus an asset in terms of the architecture complexity. For this reason, in the sequel, the second solution was selected to attenuate the strong signal.

The strong signal's carrier frequency is matched to the notch filter's central frequency by using a frequency translation. Then, the strong signal's central frequency must be computed by the digital part of the architecture (DSP) in order to set the frequency of the first local oscillator $\left(L O_{1}\right)$. To do so, the entire $8 \mathrm{MHz}$ signal bandwidth must be digitized without passing through the notch filter. The frequency seeking may be done by the means of a Fast Fourier Transform (FFT) in a second branch of the architecture. On this second branch, only the strongest signal will be digitized and the ADC resolution on this branch should be set in a such a way to be able to properly digitize only one signal. In [Vallerian et al., 2014], it has been shown that a resolution of 5 bits is sufficient to perform this digitization. Therefore, in the following, the two branches of the proposed architecture are respectively referred to as fine digitization path (FDP) and coarse digitization path (CDP).

In the architecture presented in Figure 3, the top and bottom branches represent the FDP and the CDP, respectively. In the CDP, the input signals are digitized after the frequency down conversion. Then, a Digital Signal Processor (DSP) performs the FFT and finds the strong signal's carrier frequency by seeking the maximum frequency components of the spectrum. Then, it drives the Local Oscillators (LOs) of the FDP in order to synthesize this frequency.

On the FDP, the input signals are first down-converted around an intermediate frequency through the $L O_{1}$ to make the strong signal frequency match the notch filter's central frequency. The signals are down converted in baseband afterward by using the $\mathrm{LO}_{2}$ and then digi- tized. The demodulation of the two baseband signals is performed by the DSP.

The noteworthy frequencies of this approach are represented in Figure 4. $f_{R F}$ is the starting frequency of the RF $8 \mathrm{MHz}$ band. $f_{S s}$ and $f_{S w}$ are the strong and weak signal baseband frequencies, and $f_{S s, R F}$ and $f_{S w, R F}$ are the corresponding RF frequencies. $f_{L O 1}$ and $f_{\mathrm{LO} 2}$ are the $L O_{1}$ and $L O_{2}$ frequencies and $f_{c C B}$ is the notch filter central frequency. $f_{I}$ is the starting frequency of the intermediate $8 \mathrm{MHz}$ band. In the presented scenario, $f_{I}=f_{L O 2}$.

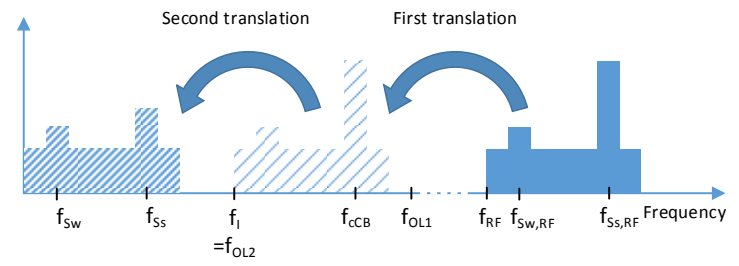

Figure 4. The noteworthy frequencies of the architecture, denoting the frequencies of the signals and the starting frequency of the band in RF, IF and BB.

To perform the frequency conversion on the FDP, $f_{L O 1}$ should be set to make $f_{S s, R F}$ to be equal to $f_{C C B}$ :

$$
f_{L O 1}=f_{S s, R F}-f_{C C B}
$$

The second frequency down conversion is intended to complete the baseband conversion initiated by LO1:

$$
f_{L O 2}=f_{R F}-f_{L O 1}
$$

\section{Simulation results}

The proposed architecture has been implemented on the Keysight's ADS software in order to study its performance. A strong and a weak signal are generated with a Binary Phase-Shift Keying (BPSK) modulation. Their power ratio is adjustable and an Additive White Gaussian Noise (AWGN) is introduced to simulate a tunable $E_{b} / N_{0}$ that is considered with respect to the weak signal. 
As a reminder, in the case of AWGN noise, the theoretical Bit-Error Rate (BER) for a BPSK modulation is:

$$
B E R=\frac{1}{2} \operatorname{erfc}\left(\sqrt{\frac{E_{b}}{N_{0}}}\right)
$$

where $\operatorname{erfc}(\mathrm{x})$ is the complementary Gauss error function of $x$.

The architecture's performance is measured as the difference between the theoretical BER and the simulated BER. The BER is simulated through the Monte-Carlo method and is given in the following with a relative variance of 0.01 .

It is assumed that the strong signal's spectrum is not spread, even if spreading is a common technique employed in urban sensor networks. Indeed this technique is used to improve the sensitivity of a technology, and is thus employed by communication protocols covering sensitive applications (whose sensors are located indoor or underground for example). As we consider that the strong signal is emitted in LOS conditions, it is assumed the employed communication protocol (covering low data rate applications) will not use such a technique. Its bandwidth is considered to be $50 \mathrm{kHz}$, which is a common value for this kind of signals (ETSI [2011]). If its bandwidth is higher, it is assumed that its power will be lower. Then a partial attenuation would still be efficient, as the strong signal should be less attenuated to reach a sustainable dynamic range. The weak signal is assumed to have a bandwidth of $200 \mathrm{kHz}$.

Despite the assumptions made (the strong signal is emitted with a small bandwidth, and only one strong signal is present on the band), the studied case is a worstcase scenario: the presence of two signals with a $100 \mathrm{~dB}$ power ratio, which is rare in practice.

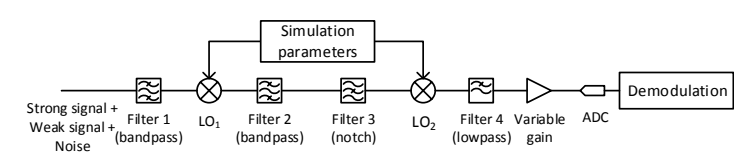

Figure 5. FDP implementation on the ADS software. The input signals are translated on the notch filter to attenuate the strongest one, and then translated in baseband to be digitized. The frequencies of LO1 and LO2 are set from the simulation parameters.

The FDP implementation is presented in Figure 5. For the sake of readability, the signals generation and demodulation are not shown in this figure. The input signals (including the strong and the weak signals and the noise) are first filtered to avoid aliasing during the $L O_{1}$ frequency translation. The second filter in the chain eliminates the signals' image frequency, and the filter 3 is the notch filter that attenuates the strong signal. After $\mathrm{LO}_{2}$ 's baseband translation, the signals then pass through the filter 4 , which is the ADC's anti-aliasing filter. They are eventually digitized and demodulated by the DSP. The notch filter's central frequency $f_{c C B}$ should be low in order to minimize the quality factor of the filter (and thus to guarantee a simpler implementation).

Because of the $8 \mathrm{MHz}$ bandwidth of the considered communication system, the intermediate band should begin at frequencies higher than $4 \mathrm{MHz}$ to avoid aliasing during the baseband translation. $f_{C C B}$ is then chosen to be $12 \mathrm{MHz}$ to be able to cut the strong signal regardless of its frequency. It is imposed that the filter should not attenuate other signals than the strong signal and its two adjacent channels. The strong signal channel being $50 \mathrm{kHz}$, the cut bandwidth should be $150 \mathrm{kHz}$. This leads to a quality factor of 80 , which is a reasonable value considering today's technology. In a first step, the signals should be sufficiently far from each other to avoid any influence from the notch filter on the weak signal. The signals' baseband frequencies are then set to be $f_{S w}=2 \mathrm{MHz}$ and $f_{S s}=5 \mathrm{MHz}$, respectively.

As mentioned, the architecture implementation is validated through the simulated BER. The BER is plotted as a function of $E_{b} / N_{0}$ in the Figure 6 . The thin solid line represents the theoretical BER, calculated for a BPSK modulation based on equation (3). The thick solid line is the simulated BER with the proposed architecture and the dashed line is the BER with a classical architecture (i.e. the same architecture without the notch filter and the intermediate band translation).

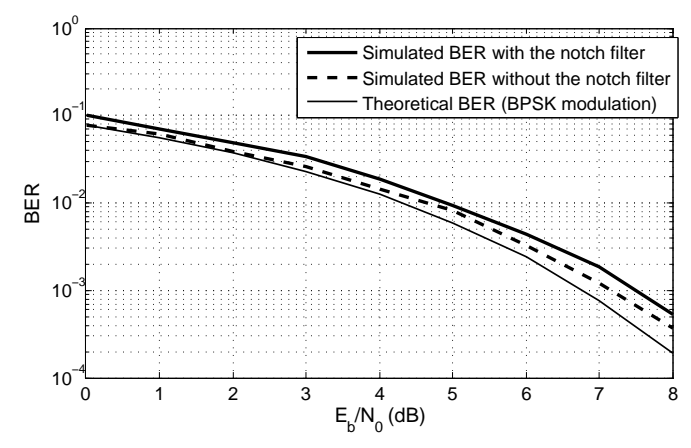

Figure 6. Simulated BER of the weak signal with the proposed implementation and with a more classical implementation. In simulations, the BER is lightly degraded with respect to the theoretical one, but they are still of same order. 
The BER with the classical architecture is a reference to which the BER with the proposed architecture is compared to. It can be seen that with a classical architecture, $E_{b} / N_{0}$ is degraded by about $0.5 \mathrm{~dB}$. This is due to the filters that lightly corrupt the signal but sufficiently to rise $E_{b} / N_{0}$. It can be noted that this degradation does not depend on $E_{b} / N_{0}$.

With the proposed architecture, $E_{b} / N_{0}$ is degraded by only $0.3 \mathrm{~dB}$ with respect to the classical architecture, because of the intermediate band frequency translation's filters. This implementation can then be used for further studies, as it provides a very small degradation with regards to a classical architecture.

The CDP is simpler to implement since no additional frequency translation is required, only one frequency translation is performed to translate the transmission band into baseband. The strong signal frequency measurement must be performed on this branch. To do so, an FFT is done and the maximum frequency component is found. quencies are set directly from the simulation parameters giving the central frequencies of each channel. This allows to isolate the different parameters and to study each of them separately, as the strong signal frequency measurement is not susceptible to be a source of error.

\subsection{Influence of the notch filter selectivity}

It has been shown in section 2 that the notch filter must provide a $40 \mathrm{~dB}$ attenuation. To reach this attenuation, the filter is tested with several orders and its bandwidth must be adapted depending on this order. The purpose of a first set of simulations is thus to give the filter bandwidth that provides a $40 \mathrm{~dB}$ attenuation for several orders. The filter order is chosen to vary from 1 to 5 , this range being a good compromise between complexity and efficiency. The simulated bandwidths are presented in Table 1 and these values are used in the following in the filter selectivity simulations.

\section{Robustness of the proposed architectyre}

Compared to the implementation presented in the previous section, the notch filter is changed to a cut-band filter, replacing the two bandpass filters. This implementation increases the simulation time but is more realistic.

\subsection{Overall simulation parameters}

To test the robustness of the proposed architecture, several parameters are to be considered. The notch filter should attenuate the strong signal without affecting the other ones. It was established in section 3 that the filter should have a $50 \mathrm{kHz}$ cut band and should not affect other signals in a band wider than $150 \mathrm{kHz}$. The filter order and cut bandwidth (i.e. the filter selectivity) play an important role on the architecture performance, hence several simulations are performed in order to establish their required characteristics which are not affecting the weaker signals.

Another important parameter is the oscillators accuracy. Indeed, since the oscillators' frequencies are set from the strong signal's frequency measurement through (1) and (2), a measurement error implies that the strong signal will not be translated exactly on the notch filter's central frequency and it will be only partially attenuated.

In order to study the influence of these frequencies generation accuracy on the overall architecture performance, several simulations were performed. First, the notch filter's selectivity and the signal's frequency proximity have been varied and second, the LOs frequency accuracy limits have been tested. Then, the oscillators fre-

Table 1. Notch filter attenuation on a strong signal of a $50 \mathrm{kHz}$ bandwidth, depending on the filter's order and bandwidth

\begin{tabular}{|c|c|c|}
\hline Filter order & Filter bandwidth & Strong signal attenuation \\
\hline 1 & $742 \mathrm{kHz}$ & $40.1 \mathrm{~dB}$ \\
\hline 2 & $265 \mathrm{kHz}$ & $40.1 \mathrm{~dB}$ \\
\hline 3 & $114 \mathrm{kHz}$ & $40.5 \mathrm{~dB}$ \\
\hline 4 & $94 \mathrm{kHz}$ & $39.7 \mathrm{~dB}$ \\
\hline 5 & $89 \mathrm{kHz}$ & $39.6 \mathrm{~dB}$ \\
\hline
\end{tabular}

It is considered that the frequency spacing between the signals and oscillators frequencies are fixed and the effect of the filter selectivity on the weak signal's BER is studied here. One channel spacing (i.e. $50 \mathrm{kHz}$ ) is kept between the strong and weak signals. In baseband, the strong signal begins at $1.975 \mathrm{MHz}$ and ranges to $2.025 \mathrm{MHz}$ and the band that can be affected by the filter thus ranges from $1.925 \mathrm{MHz}$ to $2.075 \mathrm{MHz}$. As for the weak signal's baseband channel, it is set to range from $1.725 \mathrm{MHz}$ to $1.925 \mathrm{MHz}$ to avoid being attenuated by the filter. In the performed simulations, $E_{b} / N_{0}$ is $7 \mathrm{~dB}$ with respect to the weak signal. The $S_{s} / S_{w}$ ratio is set to $80 \mathrm{~dB}$ instead of $100 \mathrm{~dB}$ because of the use of transient simulation which is less efficient when simulating high dynamic range signal but without any loss in the significance of these simulations.

The ADC resolution is set to 30 bits on the reference path to be high enough not to disrupt the BER measurement. A BER test is performed on the weak signal for every filter order. A filter order is thus validated if the simulated BER is equivalent to the theoretical one (which is of the order of $2 \cdot 10^{-3}$, from Figure 6). 


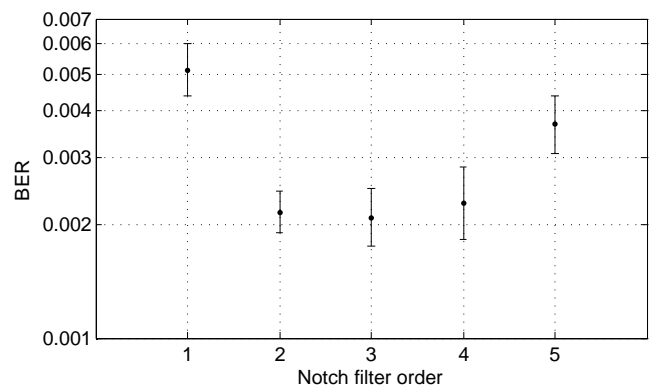

Figure 7. Simulated BER regarding the notch filter selectivity when the signals are close in frequency. The BER is degraded for the order 1 by the filter attenuation on the weak signal, and for the order 5 by the filter phase shift that affects the weak signal.

Table 2. Simulated BER regarding the notch filter selectivity

\begin{tabular}{|c|c|}
\hline Filter order & BER \\
\hline 1 & $5.11 \cdot 10^{-3}$ \\
\hline 2 & $2.15 \cdot 10^{-3}$ \\
\hline 3 & $2.08 \cdot 10^{-3}$ \\
\hline 4 & $2.27 \cdot 10^{-3}$ \\
\hline 5 & $3.67 \cdot 10^{-3}$ \\
\hline
\end{tabular}

The simulation results are presented in Table 2 and are plotted in Figure 7. In these simulations, the BER is com-

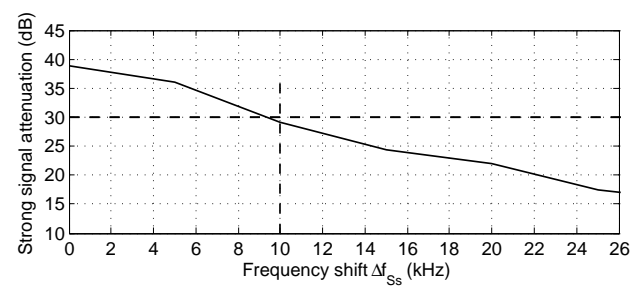

Figure 8. Strong signal attenuation regarding the measured frequency error. As the filter should have a minimum attenuation of $30 \mathrm{~dB}$, a measured frequency error less than $10 \mathrm{kHz}$ is required.

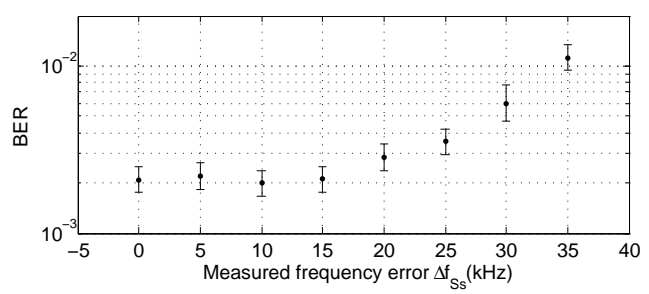

Figure 9. Simulated BER regarding the measured frequency error. The BER is degraded when the measured frequency error is higher than $15 \mathrm{kHz}$. puted with a $95 \%$ confidence interval. When the filter order is 1 , the BER is degraded because of the filter attenuation. For orders from 2 to 4 , the BER is equivalent to the theoretical one and is of the order of $2 \cdot 10^{-3}$. With an order 5 , the filter phase shift becomes too important and affects the weak signal integrity, and leads to an important BER degradation.

It is considered in the following that the order 3 is the best compromise between the non-attenuation of the weak signal and its phase shift.

4.3. Influence of the local oscillator's frequencies accuracy

In the final receiver architecture, the oscillators' frequencies are set from a measurement of the strong signal's carrier frequency. Thus they are susceptible to be inaccurate because of a measurement error. To simulate the effect of the oscillators' frequencies accuracy, an error $\Delta f_{S s}$ is introduced on $f_{S s}$ in the calculation of $f_{O L 1}$ and $f_{O L 2}$ (defined by equations (1) and (2)), which are still set from the simulation parameters (and not from a measurement on the signals). So the strong signal is translated on the filter's central frequency with a small frequency shift $\Delta f_{S s}$. This shift is canceled during the baseband conversion, so it does not affect the weak signal demodulation.

The filter order is set to 3 and the signals frequencies are set to $1 \mathrm{MHz}$ for the weak signal and $2 \mathrm{MHz}$ for the strong signal, so the filter selectivity cannot affect the weak signal. In the intermediate band, the strong signal is now translated on the frequency $f_{c C B}-\Delta f_{S s}$. Then, the filter is less efficient to attenuate the strong signal. A series of BER simulations are performed to find the oscillator's frequencies accuracy requirement to sufficiently attenuate the strong signal and to properly demodulate the weak signal. A simulated BER close to the theoretical one means that the filter properly attenuates the signal.

The architecture should be capable of digitizing an input $80 \mathrm{~dB}$ power ratio with 12 bits, since the targeted resolution improvement is 7 bits. In Figure 2, one can see that such a resolution corresponds to a power ratio of about $50 \mathrm{~dB}$. Thus the filter attenuation should be at least $30 \mathrm{~dB}$.

A series of simulations intends to evaluate the strong signal attenuation depending on the frequency shift and the results are represented in Figure 8. As can be seen, to guarantee a higher attenuation than $30 \mathrm{~dB}$ (i.e. a rejection of the strong signal of more than $30 \mathrm{~dB}$ ), the frequency measurement error should be less than $10 \mathrm{kHz}$. The shift between the attenuation at $\Delta f_{S s}=0 \mathrm{kHz}$ and the corresponding attenuation given in Table 1 is due to 
the BER measurement tolerance range. Then the BER should be simulated for this error range. The simulated BER is plotted in Figure 9, where the BER is represented as a function of the error on the $f_{S s}$ measurement. Due to the confidence interval, the error domain in which the weak signal is properly demodulated cannot be precisely bounded; however it can be estimated to be between 0 and $15 \mathrm{kHz}$. Thus, the measurement error on $f_{S s}$ should be kept in this interval.

\section{Conclusion}

In this paper, a dual branch receiver architecture capable of processing high dynamic range signals and thus relaxing the digitizer constraints is proposed. The proposed architecture assesses a problem commonly encountered in urban sensor networks in particular and in the IoT communications in general, which is the simultaneous reception of signals with very different power levels.

The architecture has been implemented on Keysight's ADS software to evaluate its feasibility. The simulations results show that the notch filter implementation is not constraining, since a filter of order 3 is enough to properly attenuate the strong signal without affecting the neighbor channels.

Moreover, the robustness related to the strong signal carrier frequency drift has been simulated. The results are quite satisfying, since a precision of $15 \mathrm{kHz}$ on a $50 \mathrm{kHz}$ channel is required. This means that some drift can be accepted on the frequency measurement.

The proposed architecture is then an efficient way to reduce the $\mathrm{ADC}$ requirements when digitizing multiple signals in urban sensor networks. Globally, instead of using a 21 bits $\mathrm{ADC}$, the proposed architecture uses two branches, one with a 5 bit ADC, for the strongest signal and one with a 14 bit ADC for all other signals. When taking into account the RF architecture impairments, the required ADC's resolution on the fine digitization path increases from 14 to 16 bit. Future work will focus on testing its performance in a more realistic case, by using real signals emitted by SRDs.

A further study will be done on the best way to perform the frequency measurement of the strong signal. In this paper it has been assumed that an FFT was used but it could also be done using a filter bank for a faster measurement.

Another work to be done on this architecture is to study its sensitivity and non-linearity requirements, as they are very important regarding the high dynamic range the architecture is intended to receive.

\section{Acknowledgment}

This work has been funded by an Orange Labs research grant and the authors wish to thank Orange Labs research team for fruitful discussion and for their collaboration in this project. The authors declare that they have no competing interests. Further additional data cannot be made publicly available.

\section{References}

Borremans, J., G. Mandal, V. Giannini, B. Debaillie, M. Ingels, T. Sano, B. Verbruggen, and J. Craninckx (2011), A $40 \mathrm{~nm}$ CMOS 0.4-6 GHz Receiver Resilient to Out-of-Band Blockers, J. Solid-State Circuits, 46(7), 1659-1671.

Dardaillon, M., K. Marquet, T. Risset, J. Martin, and H.-P. Charles (2014), Cognitive radio programming survey, in Handbook of Research on Software-Defined and Cognitive Radio Technologies for Dynamic Spectrum Management, edited by N. Kaabouch and W.-C. Hu, IGI Global.

ETSI (2011), Electromagnetic compatibility and Radio spectrum Matters (ERM); System Reference document (SRdoc): Spectrum Requirements for Short Range Device, Metropolitan Mesh Machine Networks (M3N) and Smart Metering (SM) applications, TR 103055.

Fletcher, A., S. Rangan, V. Goyal, and K. Ramchandran (2007), Robust predictive quantization: Analysis and design via convex optimization, Selected Topics in Signal Processing, IEEE Journal of, 1(4), 618-632, doi:10.1109/JSTSP.2007.910622.

Gantz, J., and D. Reinsel (2012), The digital universe in 2020: Big data,bigger digital shadows, and biggest growth in the far east, IDC iView : IDC Anal. Future, 2007, 1-16.

Jonsson, B. (2010), A survey of A/D-Converter performance evolution, in Electronics, Circuits, and Systems (ICECS), 2010 17th IEEE International Conference on, pp. 766-769, doi:10.1109/ICECS.2010.5724625.

Krzymien, L., and C. Schlegel (2011), Near-Far Resistant MIMO Iterative Receiver for Uplink LTE, in Communications (ICC), 2011 IEEE International Conference on, pp. 15, doi:10.1109/icc.2011.5963041.

Nie, H., A. Salkintzis, and P. Mathiopoulos (1999), A new approach for mitigating A/D conversion requirements in software radio base stations, in Vehicular Technology Conference, 1999. VTC 1999 - Fall. IEEE VTS 50th, vol. 5, pp. 2810-2814 vol.5, doi:10.1109/VETECF.1999.800299.

Vallerian, M., G. Villemaud, B. Miscopein, T. Risset, and F. Hutu (2014), SDR for SRD: ADC specifications for reconfigurable gateways in urban sensor networks, in Radio and Wireless Symposium (RWS), 2014 IEEE, pp. 178-180, doi:10.1109/RWS.2014.6830084.

van den Heuvel, J., and D. Cabric (2010), Spatial filtering approach for dynamic range reduction in cognitive radios, in Personal Indoor and Mobile Radio Communications (PIMRC), 2010 IEEE 21st International Symposium on, pp. 2657 -2662, doi:10.1109/PIMRC.2010.5671790. 
Wang, S., S. Kim, L.-H. Sun, H. Kim, S. Lee, S. R. Subramanya, K. Kim, and B. Yi (2005), Blind adaptive multiuser detection, in Global Telecommunications Conference, 2005. GLOBECOM '05. IEEE, vol. 6, pp. 5 pp.-3737, doi: 10.1109/GLOCOM.2005.1578468.

Corresponding author: Florin Huţu, CITI laboratoy, 6 av. des Arts, 69621, Villeurbanne, France; florin-doru.hutu@insalyon.fr

(Received 